\title{
Transverse electric current induced by optically injected spin current in a cross-shaped InGaAs/InAlAs system
}

\author{
Jian Li, ${ }^{\text {a) }}$ Xi Dai, Shun-Qing Shen, and Fu-Chun Zhang \\ Department of Physics and Centre of Theoretical and Computational Physics, \\ The University of Hong Kong, Hong Kong, China
}

(Received 3 January 2006; accepted 6 March 2006; published online 18 April 2006)

\begin{abstract}
We examine the electric response of a linearly polarized light normally shed on a cross-shaped quasi-two-dimensional InGaAs/InAlAs system with structure inversion asymmetry. The photoexcited conduction electrons carry a pure spin current with in-plane spin polarization due to the Rashba spin-orbit interaction. We use the Landauer-Büttiker formalism to show that this spin current induces two inward or outward transverse charge currents, which are observable in experiments. This effect may serve as an experimental probe of certain types of spin current. (C) 2006 American Institute of Physics. [DOI: 10.1063/1.2196230]
\end{abstract}

Spin-coherent transport of conduction electrons in semiconductor heterostructures is currently an emerging subject due to its possible application in a new generation of electronic devices. ${ }^{1}$ There have been considerable efforts to achieve spin-polarized current or pure spin current in semiconductors such as injection from ferromagnetic contact, ${ }^{2}$ quantum spin pump, ${ }^{3}$ spin Hall effect, ${ }^{4}$ and spin-like Andreev reflection. ${ }^{5}$ Optical injection of spin current is based largely on the fact that the spin-polarized carriers in conduction band can be injected in semiconductors via absorption of the circularly or linearly polarized light. ${ }^{6,7}$ While there are successful ways to inject or generate spin current, its detection is still a subtle problem. Despite the optical methods that indicated spin accumulation due to the spin current, ${ }^{8}$ it is tempting to probe spin current by measuring its electric effects. ${ }^{9-12}$ In this letter, we theoretically study electric transverse current driven by an optically injected spin current in a two-dimensional (2D) electron gas of InGaAs/InAlAs with structure inversion asymmetry. A linearly polarized light pumps electrons from valence to conduction bands, which induces a pure spin current with in-plane polarization due to the spin-orbit coupling. The Hall effect related to this spin current in a cross-shaped mesoscopic system is explored, which yields two measurable inward or outward electric transverse currents. By using the Landauer-Büttiker formalism, we provide an estimate of the electric transverse current measurable in experiments.

We begin with the optical injection of spin current in quasi-2D InGaAs/InAlAs with structural inversion asymmetry. The low-energy band structure is well known and is plotted schematically in Fig. 1(a). The conduction electron can be described by an effective Hamiltonian,

$$
H_{\text {conduction }}=\frac{\mathbf{p}^{2}}{2 m_{c}}-\frac{\alpha}{\hbar}(\mathbf{p} \times \sigma) \cdot \hat{z}+V(z),
$$

where $\sigma$ are the Pauli matrices, $V(z)$ is the asymmetric confining potential perpendicular to the sample $(x-y)$ plane, and $\alpha$ is the strength of the Rashba spin-orbit coupling. The zerofield splitting of the conduction band arises because of the Rashba coupling, and electrons are spin polarized normal to the momentum in each subband, as shown in Fig. 1(b). The

\footnotetext{
${ }^{a)}$ Electronic mail: lijian@hkusua.hku.hk
}

electrons in the valence band near the $\Gamma$ point can be described by the Luttinger Hamiltonian,

$$
H_{\text {valence }}=-\left(\gamma_{1}+\frac{5}{2} \gamma_{2}\right) \frac{\mathbf{p}^{2}}{2 m_{e}}-2 \gamma_{2} \frac{(\mathbf{p} \cdot \mathbf{S})^{2}}{2 m_{e}}+V(z),
$$

where $m_{e}$ is the free electron mass, $\gamma_{1}$ and $\gamma_{2}$ are two KohnLuttinger parameters, which are taken to be $\gamma_{1}=7.0$, and $\gamma_{2}=1.9$ in this calculation, ${ }^{13} \mathbf{S}$ represents three $4 \times 4$ spin$3 / 2$ matrices. For simplicity, we approximate $V(z)$ in Eqs. (1) and (2) by an infinite potential wall with a width of $d=10 \mathrm{~nm}$. The wave functions for the holes in the valence band can be obtained by a truncation method. We diagonalize $H_{\text {valence }}$ in a truncated Hilbert space only including the lowest $N$ basis states. In the present paper, we take $N=80$, which is accurate enough for the lowest four hole subbands. ${ }^{13}$

The process of optical excitation is schematically illustrated with the e1 and hh1 subbands in Fig. 1. When a linearly polarized light is shed normally onto the sample plane, the electrons are pumped from the hh1 subband of the valence band to the e1 subband of the conduction band via direct optical absorption provided that the photon energy is higher than the band gap, i.e., $\hbar \omega>E_{g}$. Due to the Rashba spin-orbit coupling, the conduction bands are split into two

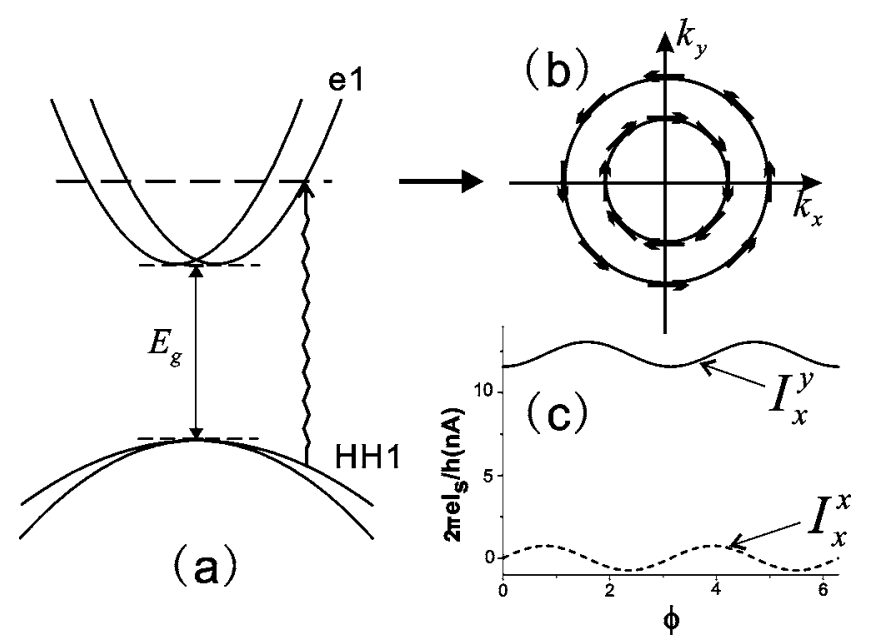

FIG. 1. (a) A schematic view of the 2D band structure of InGaAs/InAlAs. (b) Schematic plot of spin polarization (arrow) of conduction electron in momentum space with the same energy. (c) Calculated spin current induced by linearly polarized light. 
subbands, as shown in Figs. 1(a) and 1(b). A photoexcited electron has an in-plane spin polarization perpendicular to its momentum, which induces a pure spin current. The spin current operators for electrons in the conduction band and holes in the valence band can be expressed in terms of the velocity and spin operators of electrons and holes, respectively, $J_{\nu}^{\mu(\mathrm{e})}=(\hbar / 4)\left\{\sigma^{\mu}, v_{\nu}^{(\mathrm{e})}\right\}$ and $J_{\nu}^{\mu(\mathrm{h})}=(\hbar / 2)\left\{S^{\mu}, v_{\nu}^{(\mathrm{h})}\right\}$, where $\mathbf{v}^{(\mathrm{e})}(\mathbf{k})$ and $\mathbf{v}^{(\mathrm{h})}(\mathbf{k})$ are the velocity operators for the conduction and valence bands, respectively. The total spin current from the photoexcited electrons and holes is

$$
\left\langle J_{\nu}^{\mu}\right\rangle=\sum_{\mathbf{k}} \operatorname{Tr}\left\{J_{\nu}^{\mu(\mathrm{e})} \rho^{(\mathrm{e})}(\mathbf{k})-J_{\nu}^{\mu(\mathrm{h})} \rho^{(\mathrm{h})}(\mathbf{k})\right\},
$$

where $\rho^{(\mathrm{e})}(\mathbf{k})$ and $\rho^{(\mathrm{h})}(\mathbf{k})$ are the density matrices for the conduction and valence bands, respectively. The density matrices that appear in Eq. (3) can be obtained in a relaxation time approximation. In the present study only the diagonal components of the density matrices are kept, and the results can be written as ${ }^{14}$

$$
\begin{aligned}
\rho_{n n}^{(\mathrm{e}, \mathrm{h})}(\mathbf{k})= & \frac{\pi}{2 \hbar} \tau_{\mathrm{e}, \mathrm{h}} \sum_{m} M_{n m}^{*}(\mathbf{k}) M_{m n}(\mathbf{k}) \\
& \times\left[\delta\left(E_{n}^{\mathrm{e}, \mathrm{h}}(\mathbf{k})+E_{m}^{\mathrm{h}, \mathrm{e}}(\mathbf{k})-\hbar \omega_{p h}\right)\right],
\end{aligned}
$$

where $\tau_{\mathrm{e}, \mathrm{h}}$ is the relaxation time for electrons (holes) and $M$ is the $2 \times 4$ transition matrix describing the direct optical transition between the conduction and valence subbands, which is caused by the external light. Under the dipole approximation, $M$ can be expressed by $M=D_{c}^{-1} \tilde{M} D_{v}$, where $D_{c}$ and $D_{v}$ are the $2 \times 2$ and $4 \times 4$ transformation matrices that diagonalize the Hamiltonian in Eqs. (1) and (2), respectively, and the matrix $\tilde{M}$ is the coupling matrix in the original basis of the row $\left\{\left|S_{z}(=+1 / 2,-1 / 2)\right\rangle\right\}$ and column $\left\{\left|S_{z}(=+3 / 2,1 / 2,-1 / 2,-3 / 2)\right\rangle\right\}$, as

$$
\tilde{M}=\left(\begin{array}{cccc}
g e^{i \phi} & 0 & g^{*} e^{-i \phi} / \sqrt{3} & 0 \\
0 & g e^{i \phi} / \sqrt{3} & 0 & g^{*} e^{-i \phi}
\end{array}\right),
$$

where $\phi$ is the polarization angle of the linearly polarized light and the factor $g$ is determined by the Bloch functions of electron and hole at the $\Gamma$ point. In this way, the pure spin current is obtained as a function of the frequency and polarization of the light. The dominant component of the spin current flowing in the $x$ direction is $J_{x}^{y}$, which is similar to the equilibrium current proposed by Rashba. ${ }^{15}$ To calculate the spin current, we adopt the parameters from a sample of $\mathrm{In}_{x} \mathrm{Ga}_{1-x} \mathrm{As} / \mathrm{In}_{0.52} \mathrm{Al}_{0.48} \mathrm{As},{ }^{16}$ with the Rashba coupling strength $\alpha=6.1 \times 10^{-12} \mathrm{eV} \mathrm{m},\left(\alpha / \hbar \simeq 3 \times 10^{-4} c\right.$, where $c$ is the speed of light), the effective mass $m_{c}=0.05 m_{e}$, the incident light power is $100 \mathrm{~mW}$ with the wavelength $\lambda=880 \mathrm{~nm}$. We also extract from the experimental data ${ }^{16}$ that $\pi|g|^{2} \tau_{e} /\left(\hbar^{2} / m_{e} d^{2}\right) 2 \hbar \approx 0.78 \times 10^{-3}$, which is used in calculating the density matrices. Given a quantum well with the size $L \times L$, the induced current $I_{\nu}^{\mu}=J_{\nu}^{\mu} L$ varies approximately with $\cos 2 \phi$, as shown in Fig. 1(c) with $L=100 \mu \mathrm{m}$, the function of $I_{x}^{y}$ is fitted to be $I_{x}^{y} \approx I_{0}+I_{1} \cos 2 \phi$ with $I_{0}=12.32 \mathrm{nA}$ and $I_{1}=0.75 \mathrm{nA}$. It is also noticeable that there is also a nonvanishing $I_{x}^{x}$ component of the spin current, although it is comparatively negligible.

We now turn to investigate the consequence of applying this in-plane polarized spin current to a cross-shaped mesoscopic system with the Rashba spin-orbit coupling, and it

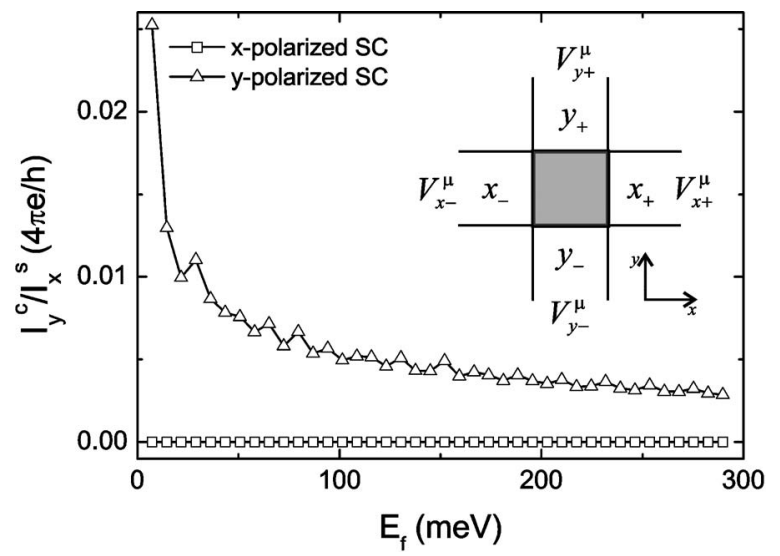

FIG. 2. The ratio of the electric transverse current to the longitudinal spin current as a function of the Fermi energy counted from the bottom of the conduction band, with inset the geometry of the cross-shaped structure in our calculation. The calculation was carried out on a $40 \times 40$ square lattice with the total width $L=100 \mathrm{~nm}$, the effective electron mass $m^{*}=0.05 m_{\mathrm{el}}$, and the Rashba spin-orbit coupling strength $\alpha=6.1 \times 10^{-12} \mathrm{eV} \mathrm{m}$.

turns out that electric transverse currents will be induced in this case. We analyze this first from the symmetry point of view, followed by an numerical estimation of the amplitude of the transverse currents, then we propose an experiment for the observation. In our proposal the whole setup is carved on an InGaAs/InAlAs heterojunction with a central scattering region and four leads for measurements, as shown in the inset of Fig. 2. The transverse leads (leads $y_{+}$and $y_{-}$) and the scattering region should be masked to prevent from the light shining explicitly, while the longitudinal leads (leads $x_{+}$and $\left.x_{-}\right)$are opened to accept the linearly polarized light to generate the incident in-plane-polarized spin current. By avoiding any possible interfaces of current injection, the spin current is expected to circulate in the $x$ direction without conventional spin injection problem. Under the LandauerBüttiker formalism, ${ }^{17,18}$ which has been extensively used in the study of quantum transport in mesoscopic systems, ${ }^{19,20}$ and will be applied to analyze the transverse currents in our case, we simulate the generation of the spin current and the measurement of the transverse currents by assuming a proper setting of the spin-dependent chemical potential related to each lead. In detail, if we denote the effective voltage related to spin polarization $\mu(\mu=\uparrow, \downarrow)$ at lead $p$ by $V_{p}^{\mu}$, then we assume $V_{x-}^{\uparrow}=-V_{x-}^{\downarrow}=-V_{x+}^{\uparrow}=V_{x+}^{\downarrow}=V_{0} / 2$ and $V_{y-}^{\uparrow}=V_{y-}^{\downarrow}=V_{y+}^{\uparrow}$ $=V_{y+}^{\downarrow}=0$. It should noted that $\mu$ can be oriented in the $x, y$, or $z$ direction (to consider either in-plane- or perpendicular-toplane-polarized spin current), which will be denoted by $\mu \sim x, y$, or $z$. Given this voltage setting, the symmetry properties of the currents will be fully presented by the spindependent transmission functions between the leads, ${ }^{20}$ as we will see.

In each lead (assumed to be ideal and semi-infinite by convention) attached to the central shaded region in the inset of Fig. 2, the wave function can be expanded in terms of separate propagating modes, which are the eigenstates in the lead. In $x_{-}$, for example, the eigenstates are $\psi_{m \mu}^{ \pm}(x, y)$ $=C e^{ \pm i k_{m} x} \phi_{m}(y) \otimes \chi_{\mu}$, where $C$ is the normalization constant, $\phi_{m}(y)$ is the $m$ th eigenstate in the transverse dimension, $\chi_{\mu}$ is the spin eigenstate with $\mu=\uparrow$ or $\downarrow$, and \pm denotes the mode as incoming or outgoing. The expansion coefficients of the actual wave functions in terms of these eigenstates are known as the wave amplitudes $a_{m \mu}^{\text {in/out }}$, which are related by an to AlP license or copyright, see http:I/lapl.aip.org/apl/copyright.jsp 
unitary matrix $a_{m \mu}^{\text {out }}=\Sigma_{n, \nu} s_{m n}^{\mu \nu} a_{n \nu}^{\text {in }}$ and all the symmetries of the transport properties in the system are embedded in this S-matrix. For simplicity, we assume that the quantum modes in opposite leads are symmetrical, which means that for each mode with wave vector $k_{m}$ and spin polarization $\mu$ in lead $x_{-}\left(y_{-}\right)$, there is a mode with the same wave vector and spin polarization in lead $x_{+}\left(y_{+}\right)$, and vice versa. In the clean limit, the Hamiltonian with the Rashba spin-orbit coupling term is obviously invariant under three unitary transformations, i.e., $H$ commutes with the time-reversal operator $T=-i \sigma_{y} K$, where $K$ is the complex-conjugate operator, and two combined operators $\sigma_{x} R_{x}$ and $\sigma_{y} R_{y}$, where $R_{x}\left(R_{y}\right)$ denotes the mirror reflection operator transforming $x \rightarrow-x \quad(y \rightarrow-y)$. While the transformed eigenstates remain as eigenstates of the original Hamiltonian, some of the amplitudes are transformed in the following ways: under the transformation of $T$,

$$
a_{m \mu}^{\text {in }} \rightarrow\left(a_{m \bar{\mu}}^{\text {out }}\right)^{*} ; \quad a_{m \mu}^{\text {out }} \rightarrow\left(a_{m \bar{\mu}}^{\text {in }}\right)^{*} ;
$$

under the transformation of $\sigma_{i} R_{i}$,

$$
\begin{aligned}
& a_{m \mu}^{\text {in(out })} \rightarrow a_{\bar{m} \mu}^{\text {in }(\text { out })} \quad\left(m \in i_{-}, i_{+} ; \mu \sim i\right) \\
& a_{m \mu}^{\text {in(out })} \rightarrow a_{\bar{m} \bar{\mu}}^{\text {in }(\text { out })} \quad\left(m \in i_{-}, i_{+} ; \mu \sim j \neq i\right) ;
\end{aligned}
$$

where $i=x$ or $y, \bar{\mu}=-\mu$ and $\bar{m}$ is the counterpart of $m$ in its opposite lead. The phase factors are neglected in the above transformations because they will not be manifested in the following calculations of the transmission probabilities. The symmetries as well as the unitary condition impose constraints on the S-matrix, and thus on the transmission probability from mode $\{n, \nu\}$ to mode $\{m, \mu\}$, which is defined as $T_{m n}^{\mu \nu} \equiv\left|s_{m n}^{\mu \nu}\right|^{2}$. For the time reversal symmetry $T$, this yields

$$
T_{m n}^{\mu \nu}=T_{n m}^{\bar{\nu} \bar{\mu}}
$$

for the symmetry under $\sigma_{i} P_{i}$,

$$
\begin{aligned}
& T_{m n}^{\mu \nu}=T_{m^{\prime} n^{\prime}}^{\mu \nu} \quad(\mu, \nu \sim i) \\
& T_{m n}^{\mu \nu}=T_{m^{\prime} n^{\prime}}^{\bar{\mu} \bar{\nu}} \quad(\mu, \nu \sim j \neq i),
\end{aligned}
$$

where $i=x$ or $y, m^{\prime}=\bar{m}$ if $m \in i_{-}, i_{+}$, or $m$ otherwise. By summing up all the transmission probabilities between two leads with specific spin polarizations, the transmission functions $\bar{T}_{p q}^{\mu \nu}=\sum_{m \in p, n \in q} T_{m n}^{\mu \nu}$, and the currents are obtained using the extended Büttiker formula ${ }^{17} I_{p}^{\mu}=(e / h) \Sigma_{q, \nu}\left(\bar{T}_{p q}^{\mu \nu} V_{q}^{\nu}\right.$ $\left.-\bar{T}_{q p}^{\nu \mu} V_{p}^{\mu}\right)$ with the electric and spin currents defined as $I_{p}^{c}=e\left(I_{p}^{\uparrow}+I_{p}^{\downarrow}\right)$ and $I_{p}^{s}=(\hbar / 2)\left(I_{p}^{\uparrow}-I_{p}^{\downarrow}\right)$, respectively. Combining the symmetry-derived Eqs. (8) and (9) with the preceding voltage configuration, we find

$$
\begin{aligned}
& I_{y-}^{c}=-I_{y+}^{c}=0, \quad(\mu \sim x), \\
& I_{y-}^{c}=I_{y+}^{c}, \quad(\mu \sim y), \\
& I_{y-}^{c}=-I_{y+}^{c}, \quad(\mu \sim z) .
\end{aligned}
$$

It is clear now to see the difference between the transverse electric currents induced by a z-direction-polarized spin current and an in-plane-polarized spin current, that is in the former case the transverse current is a truly circulating one, which can be naturally regarded as a reversed effect of the spin Hall effect, ${ }^{9-11}$ whereas in our case of in-plane polariza- tion, the transverse currents are flowing both inward or outward instead of circulating through the two transverse leads. Consequently, this will make an essential difference in the measurements of the currents in such systems. To present a quantitative estimate of the induced currents, we make numerical calculations with the tight-binding approximation in this cross-shaped mesoscopic system, and the ratio of the induced electric transverse current $I_{y}^{c}$ to the spin current $I_{x}^{s}$ are plotted in Fig. 2. Combined with the calculated value of the injected spin current, we notice that the induced current is about $0.1 \sim 0.2 \mathrm{nA}$, which is large enough to be measured experimentally, while the small part of the spin current with spin polarization along the $x$ axis does not contribute to the transverse currents.

All parameters in our calculation are adopted from a realistic sample of InGaAs/InAlAs heterojunction. Thus, the effect may serve as an experimental probe of in-planepolarized spin currents.

The authors thank J. Sinova for helpful discussions. This work was supported in part by the RGC of Hong Kong under Grant No. HKU 7039/05P.

${ }^{1}$ S. A. Wolf, D. D. Awschalom, R. A. Buhrman, J. M. Daughton, S. von Molnár, M. L. Roukes, A. Y. Chtchelkanova, and D. M. Treger, Science 294, 1488 (2001)

${ }^{2}$ A. G. Aronov, Zh. Eksp. Teor. Fiz. 71, 370 (1976) [Sov. Phys. JETP 44, 193 (1976)]; M. Johnson and R. H. Silsbee, Phys. Rev. Lett. 55, 1790 (1985); for a review, see I. Zutić, J. Fabian, and S. D. Sarma, Rev. Mod. Phys. 76, 323 (2004)

${ }^{3}$ S. K. Watson, R. M. Potok, C. M. Marcus, and V. Umansky, Phys. Rev. Lett. 91, 258301 (2003).

${ }^{4}$ S. Murakami, N. Nagaosa, and S. C. Zhang, Science 301, 1348 (2003); J. Sinova, D. Culcer, Q. Niu, N. A. Sinitsyn, T. Jungwirth, and A. H. MacDonald, Phys. Rev. Lett. 92, 126603 (2004); S. Q. Shen, M. Ma, X. C. Xie, and F. C. Zhang, Phys. Rev. Lett. 92, 256603 (2004).

${ }^{5}$ B. G. Wang, J. Peng, D. Y. Xing, and J. Wang, Phys. Rev. Lett. 95, 086608 (2005).

${ }^{6}$ S. D. Ganichev, E. L. Ivchenko, S. N. Danilov, J. Eroms, W. Wegscheider, D. Weiss, and W. Prettl, Phys. Rev. Lett. 86, 4358 (2001); S. D. Ganichev, E. L. Ivchenko, V. V. Bel'kov, S. A. Tarasenko, M. Sollinger, D. Weiss, W. Wegscheider, and W. Prettl, Nature (London) 417, 153 (2002).

${ }^{7}$ R. D. R. Bhat and J. E. Sipe, Phys. Rev. Lett. 85, 5432 (2000); R. D. R. Bhat, F. Nastos, A. Najmaie, and J. E. Sipe, ibid. 94, 096603 (2005); E. Ya. Sherman, A. Najmaie, and J. E. Sipe, Appl. Phys. Lett. 86, 122103 (2005).

${ }^{8}$ Y. K. Kato, R. C. Myers, A. C. Gossard, and D. D. Awschalom, Science 306, 1910 (2004); J. Wunderlich, B. Kaestner, J. Sinova, and T. Jungwirth, Phys. Rev. Lett. 94, 047204 (2005).

${ }^{9}$ J. E. Hirsch, Phys. Rev. Lett. 83, 1834 (1999).

${ }^{10}$ P. Zhang, J. Shi, D. Xiao, and Q. Niu, Phys. Rev. Lett. 96, 076604 (2006).

${ }^{11}$ E. M. Hankiewicz, J. Li, T. Jungwirth, Q. Niu, S. Q. Shen, and J. Sinova, Phys. Rev. B 72, 155305 (2005).

${ }^{12}$ S. Q. Shen, Phys. Rev. Lett. 95, 187203 (2005).

${ }^{13}$ X. Dai, Z. Fang, Y. G. Yao, and F. C. Zhang, Phys. Rev. Lett. 96, 086802 (2006).

${ }^{14}$ J. J. Sakurai, Modern Quantum Mechanics (Addison-Wesley, Reading, MA, 1994).

${ }^{15}$ E. I. Rashba, Phys. Rev. B 68, 241315 (2003).

${ }^{16}$ J. Nitta, T. Akazaki, H. Takayanagi, and T. Enoki, Phys. Rev. Lett. 78, 1335 (1997); C. L. Yang, T. H. He, L. Ding, L. J. Cui, Y. P. Zeng, J. N. Wang, and W. K. Ge (unpublished).

${ }^{17}$ M. Büttiker, Phys. Rev. Lett. 57, 1761 (1986).

${ }^{18}$ S. Datta, Electronic Transport in Mesoscopic Systems (Cambridge University Press, Cambridge, 1995).

${ }^{19}$ L. Sheng, D. N. Sheng, and C. S. Ting, Phys. Rev. Lett. 94, 016602 (2005); B. K. Nikolić, L. P. Zârbo, and S. Souma, Phys. Rev. B 72, 075361 (2005); E. M. Hankiewicz, L. W. Molenkamp, T. Jungwirth, and J. Sinova, ibid. 70, 241301(R) (2004); J. Li, L. B. Hu, and S. Q. Shen, ibid. 71, 241305(R) (2005)

${ }^{20}$ T. P. Pareek, Phys. Rev. Lett. 92, 076601 (2004); F. Zhai and H. Q. Xu, ibid. 94, 246601 (2005). 